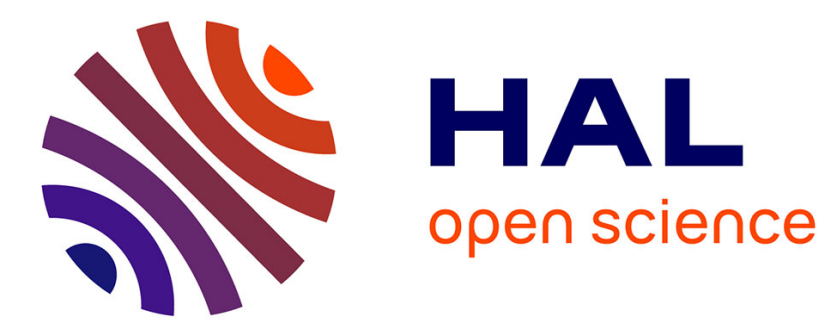

\title{
Shallow mixing layer downstream from a sudden expansion
}

\author{
Lei Han, Emmanuel Mignot, Nicolas Rivière
}

\section{To cite this version:}

Lei Han, Emmanuel Mignot, Nicolas Rivière. Shallow mixing layer downstream from a sudden expansion. Journal of Hydraulic Engineering, 2017, 143 (5), pp.04016105. 10.1061/(ASCE)HY.19437900.0001274. hal-01724987

\section{HAL Id: hal-01724987 \\ https://hal.science/hal-01724987}

Submitted on 21 Nov 2018

HAL is a multi-disciplinary open access archive for the deposit and dissemination of scientific research documents, whether they are published or not. The documents may come from teaching and research institutions in France or abroad, or from public or private research centers.
L'archive ouverte pluridisciplinaire HAL, est destinée au dépôt et à la diffusion de documents scientifiques de niveau recherche, publiés ou non, émanant des établissements d'enseignement et de recherche français ou étrangers, des laboratoires publics ou privés. 


\section{SHALLOW MIXING LAYER DOWNSTREAM A SUDDEN EXPANSION}

\author{
Lei Han ${ }^{1}$, Not a Member, ASCE \\ Emmanuel Mignot ${ }^{2}$, Not a Member, ASCE \\ Nicolas Riviere ${ }^{3}$, Not a Member, ASCE
}

\section{ABSTRACT}

The present paper aims at investigating the mixing layer located at the interface between the free stream and the recirculation zone downstream an open-channel, sudden, lateral expansion. Specific attention is paid on the interaction of the shallowness of the flow, characterized by the bed friction number, with the lateral confinement, due to the side wall. The velocity field for four flows, with the same geometry but very different bed friction numbers, is measured in detail in order to characterize the mean velocity fields and Reynolds stresses across the mixing layers and to evaluate the width of the mixing layers and their growth rates along with the typical oscillation frequencies. In the upstream region of the recirculation zone, the mixing layer characteristics for our configurations are analogous to the ones of classical - laterally unbounded - mixing layers. In this region, the shallowness modifies the shape of the streamwise velocity profiles, extends the mean velocity gradient magnitudes, lowers the Reynolds stress terms but hardly affects the mixing layer expansion rate. On the other hand, in the region near the flow reattachment, the mixing layer adopts a very different behavior, with an abrupt drop of the mixing layer expansion. This change in behavior is linked to the dynamics of the $2 \mathrm{D}$ vortices within the mixing layer. It is not due to a damping effect of the bed friction on these vortices as the local bed friction numbers remain much lower than the critical values reported in the literature. It is rather due to the interaction of the coherent structures with the side wall, the characteristics of this interaction being itself influenced

\footnotetext{
${ }^{1}$ LMFA, CNRS-Université de Lyon, INSA de Lyon, Bat. Joseph Jacquard, 20 Av. A. Einstein, 69621 Villeurbanne, France. E-mail: hanleilyon@hotmail.com.

${ }^{2}$ LMFA, CNRS-Université de Lyon, INSA de Lyon, Bat. Joseph Jacquard, 20 Av. A. Einstein, 69621 Villeurbanne, France. E-mail: emmanuel.mignot@insa-lyon.fr.

${ }^{3}$ LMFA, CNRS-Université de Lyon, INSA de Lyon, Bat. Joseph Jacquard, 20 Av. A. Einstein, 69621 Villeurbanne, France. E-mail: riviere.nicolas@insa-lyon.fr.
} 
by the flow shallowness. We additionally show that the damping effect due to bed friction is not responsible for the huge variations reported on the recirculation zones length. This imposes to distinguish between a "local mixing-layer shallowness" - derived using the mixing layer width as length scale and governing the mixing layer characteristics - and a "global flow shallowness" derived using the expansion width and governing the recirculation length.

Keywords: Shallow, Mixing layer, Recirculation zone, Backward facing step, Vortex .

\section{INTRODUCTION}

As a flow separates from a side wall, a recirculation zone forms until the flow reattaches to the wall further downstream. Li and Djilali (1995) listed the most common geometries giving birth to a recirculation zone including upward and backward facing steps, sudden expansions or flows around obstacles such as cylinders or plates. Within the recirculation zone, the net discharge is nil with limited positive streamwise velocities near the separating streamline and negative reverse velocities near the side wall. In natural streams, these regions have a major implication for transport of scalar or sediment (O'Connor et al. (2010) and Engelhardt et al. (2004)) as the limited velocities make the recirculation a privileged zone of material deposition. In the literature dealing with recirculation zones, most attention is paid to the prediction of the recirculation length, i.e. the location of the reattachment point (Li and Djilali (1995)).

On the other hand, along the interface between the free stream and the recirculation zone, the strong transverse gradient of streamwise velocity leads to a vertical mixing layer, defined by Pope as "a turbulent flow that forms between two uniform, nearly parallel streams of different velocity" (Pope (2008)). A review of the most studied mixing layers configurations in open-channel flows under deep conditions was established by Mignot et al. (2014a) and Mignot et al. (2014b). These configurations exhibit the following behavior: the width of the mixing layer increases along the streamwise axis from up- to downstream with the occurrence of advected turbulent eddies and a maximum turbulence production occurs along this mixing layer due to the increased shear and velocity gradient. These terms are thus maximum along the centerline of the mixing layer and rapidly decrease on both sides, while their magnitude decreases towards downstream. Nevertheless, the 
mixing layer considered herein develops at the interface between a main stream and a recirculation zone downstream from a shallow sudden expansion (SSE). This mixing layer is not free as it exhibits both lateral confinement due to the side wall and vertical confinement due to the impact of the bed.

\section{Effect of the lateral confinement}

Among the geometries exhibiting a recirculation, the open-channel sudden lateral expansions and the backward facing steps appear as similar configurations a priori with a similar lateral confinement. In both cases, the walls upstream and downstream from the step are parallel to each other and the flow section suddenly increases towards downstream. The recirculation zone is thus constrained by two perpendicular walls and closed along the third side by the separating streamline. Studies describing the characteristics of the separating mixing layer downstream backward facing steps show that (see Chandrsuda and Bradshaw (1981), Jovic and Driver (1994), Kasagi and Matsunaga (1995)) the axis of the mixing layer just downstream the separation is parallel to the inflow while further downstream, the mixing layer approaches the downstream wall with an increasing angle. As a consequence, in the upstream part of the recirculation the mixing layer strongly resembles typical free mixing layers (see Bell and Mehta (1990) or Wygnanski and Fiedler (1970)) with a maximum turbulent activity occurring along the centerline of the mixing layer. Further downstream, the mixing layer becomes affected by the side wall and the location of maximum Reynolds stress and turbulent production is shifted from the side wall at a distance which is not discussed in details in the papers cited above, but will be analyzed in the present paper.

\section{Effects of the vertical confinement}

The mixing layer which occurs in the open-channel sudden lateral expansions was far less investigated than the one in backward facing steps. The main difference is the vertical confinement due to the limited water depth encountered in the expansion along the direction perpendicular to the recirculation plane. A first effect (Uijttewaal and Booij (2000)) is that, with this geometrical restriction of the water column, "eddies with dimensions larger than the water depth can only move in the horizontal plane. The limited depth prohibits these eddies to be stretched in the ver- 
tical direction and forces the large eddies into a quasi two-dimensional motion." Yet, the mixing layer growth is connected to the behavior of the $2 \mathrm{D}$ coherent structures it contains. The dimensions of these structures, generated in the upstream region of the mixing layer, increase towards downstream (Uijttewaal and Booij (2000); Loucks and Wallace (2012)). However, the flow is additionally confined between a stick condition at the bed and a zero vertical but free horizontal slip velocity at the free-surface. This confinement creates a strong vertical gradient of velocity and thus additional turbulence generated in the near wall region (Babarutsi et al., 1989). As a consequence, the shallowness causes a limitation of the growth of the mixing layer width along its development: while this growth rate is constant along the streamwise axis for a deep mixing layer, in shallow conditions, the growth rate equals that of the deep conditions in the upstream region but it then decreases when advancing towards downstream (see Uijttewaal and Booij (2000)).

To characterize the shallowness of the flow (i.e. the vertical confinement), a bed friction number $S$ (also referred in the literature as "stability parameter") was introduced by Chu et al. (1983) based on a linear stability analysis of the depth-averaged shallow water equations and later by Chu et al. (1991) based on the turbulent kinetic energy analysis of the same equation where a perturbation is introduced. This coefficient characterizes the ratio between the stabilizing effect due to the bed friction (through dissipation of turbulent kinetic energy) and destabilizing effects due to the transverse shear along the mixing layer (through turbulent kinetic energy production).

In simple shallow mixing-layer configurations (see Sukhodolov et al. (2010), Uijttewaal and Booij (2000), Van Prooijen and Uijttewaal (2002)) corresponding to two parallel inflows with different velocities suddenly released on the side of each other, the linear stability analysis proved the existence of a critical bed friction number (Chu et al. (1983)), noted $S_{c}$ so that: i) in shallow conditions, $S$ exceeds $S_{c}$ and the bottom friction impedes the growth of instabilities while ii) in deep conditions $S$ remains lower than $S_{c}$ and the bottom friction has negligible effect on the growth of instabilities. Chu et al. (1983) propose $S_{c} \sim 0.12$ through an inviscid theory while Alavian and Chu (1985) found $S_{c}=0.06$ by additionally considering the turbulent motions and later Chu et al. (1991) obtained $S_{c} \sim 0.12-0.145$. Based on measurements of the growth rate of 
the shallow mixing layer width, Chu and Babarutsi (1988) found $S_{c}=0.09$ while Uijttewaal and Booij (2000) obtained $S_{c}=0.08$. However, Van Prooijen and Uijttewaal (2002)) cast doubt on the concept of a critical bed friction number itself. Indeed, the existence of a critical value $S_{c}$ is obtained by considering the most unstable mode in the linear stability. These authors consider instead the stability of the dominant mode, i.e. of the wave number corresponding to the maximum energy density or, in other words, corresponding to the coherent structures taking place in the flow. Instead of the two regimes listed above ( $S_{c}$ acting as the threshold), Van Prooijen and Uijttewaal (2002) consider three regimes: (i) growth of the dominant mode, (ii) decay of the dominant mode with other modes still growing and (iii) decay of all modes for $S>S_{c}$. These three regimes are well reproduced by their linear stability analysis, when compared with experimental measurements and can explain the scattering values of $S_{c}$ previously reported in the literature $\left(S_{c}=0.06-0.145\right.$, see above). Unfortunately, Van Prooijen and Uijttewaal (2002)'s analysis requires a function fitting the transverse velocity profile along the flow, which is unavailable for the present sudden expansion configuration. Thus, as the concept of critical bed friction number provides a fair overview of the behaviour of the shallow mixing layer, regardless of the scattering on the value of $S_{c}$, it will be used hereafter to identify our mixing layer regime, as still done in recent contributions for vertically confined mixing layers by Constantinescu (2013). In the following, we thus consider that the shallowness stabilizes the mixing layer if the local bed friction number reaches $\mathrm{O}(0.1)$.

\section{Specific case of the shallow sudden lateral expansion SSE}

To the authors' knowledge, the only analyses of the mixing layers developing downstream a SSE were performed, using experimental approach, by Babarutsi et al. (1989) using a 1D velocity probe along the streamwise axis and more recently by Talstra (2011) through Surface-PIV. These studies show that the SSE mixing layers exhibit high similarities with the more classical mixing layers: notably, the streamwise velocity fluctuation is maximum along the separating streamline and decreases on both sides. The main differences between a laterally-free mixing layer and a SSE mixing layer are that for a SSE: the velocity on the slow flow side (i.e. in the recirculation zone) is quite negligible and the curvature of the mixing layer centerline increases towards downstream, 
until reaching the reattachment point.

Babarutsi et al. (1989) propose to extrapolate this knowledge at the local, mixing layer, scale to predict the length $L$ of the recirculation zone downstream a SSE (see Fig.1) at the integral scale. With this purpose, Babarutsi et al. (1989) adopt as integral transverse length scale the expansion width $(d)$ and as vertical length scale the water depth at the expansion section $\left(h_{0}\right)$, as depicted in Fig.1. The (integral) bed friction number, noted $S_{d}$, finally reads:

$$
S_{d}=\frac{c_{f 0} d}{2 h_{0}}
$$

with $c_{f 0}$ the friction coefficient at the expansion section. Using again the concept of critical bed friction number, Babarutsi et al. (1989) define two flow regimes separated by a critical value of the integral bed friction number $S_{d}=0.05-0.1$ : for $S_{d}<0.05$ the flow is referred to as « deep water flow » and the relative recirculation length is $L / d=8$; for $S_{d}>0.1$ the flow is called a « shallow water flow » and the relative recirculation length is $L / d=0.6 / S_{d}$. Later, Chu et al. (2004) instead name these two regimes, respectively, «non-frictional » and « frictional » modes, and, with additional experimental results, propose $L / d=0.7 / S_{d}$ for the shallow water flows (frictional mode).

Through recent experiments in the Laboratory of Fluid Mechanics and Acoustics (LMFA) at the University of Lyon (INSA-Lyon, France), Chatelain et al. (2014) observe quite a different behavior depicted by Fig. 2 that plots a series of 16 non-dimensional recirculation lengths configurations with increasing $S_{d}$ values with $R_{b}=(B-d) / B=0.75$. The "bell" shaped curve on Fig.2 differs from the two asymptotic regimes proposed by Babarutsi et al. (1989) or Chu et al. (2004): it indeed exhibits a maximum non-dimensional length $L / d$ for $S_{d} \sim 0.01$ and decreasing lengths both for lower and higher $S_{d}$ values. However, this work confirms that the macroscopic (integral) behavior of the recirculating zone (typically its length) is a function of the integral bed friction number $S_{d}$.

Talstra (2011)'s study explores experimentally the classical mixing layer features (velocity profiles, Reynolds stress profiles, mixing layer width, coherent structures with the mixing layer) for three different SSE mixing layers. It shows that the initial mixing layer growth is similar to the one for a free mixing layer and that interactions exist between the main recirculation and the vortex 
shedding. Numerical simulations supplement the experiments to explore the role of secondary currents and of upstream disturbances. However, the expansion ratio $R_{b}$ varies from one experiment to another while this parameter was shown to influence the flow downstream the expansion (Riviere et al. (2008) and Chatelain et al. (2014)). This prevents from concluding regarding the influence of the shallowness alone on the SSE flow.

\section{Scientific issue}

To summarize, it appears firstly that 1) laterally bounded deep mixing layers and 2) laterally unbounded shallow mixing layers are well described in the literature. Laterally bounded deep mixing layers studies report 3D turbulence with emphasis on the reattachment point at the wall. Shallow mixing layers studies report 2D turbulence with emphasis on the influence of the shallowness on the growing rate of the mixing layer width. Conversely, little attention was devoted to the "combined" case of both laterally bounded and shallow mixing layers as the one occurring in the shallow open-channel sudden lateral expansions. In particular, the combined effects of the shallowness and of the lateral wall on the mixing layer are expected to interact with each other at the reattachment point and this interaction is still to be studied. Secondly, two parameters are used in the literature to quantify the shallowness. The local bed friction number $S$ uses the mixing layer width as horizontal length scale and governs the evolution of the mixing layer width and of the associated 2D large scale turbulent structures. The integral bed friction number $S_{d}$ uses the expansion width as length scale and characterizes the recirculation length. The connection of $S_{d}$ to $S$, i.e. the connection of the recirculation length to the adjacent mixing layer, remains to be confirmed.

Therefore, the first objective of the present work is to measure and characterize the shallow mixing layer downstream a sudden expansion and then to emphasize its differences with more simple configurations in order to finally sort the combined effects of the shallowness and of the lateral confinement on the mixing layer, especially in the region of the reattachment point. The second objective is to examine if the phenomena at the mixing layer scale (notably the coherent structures behavior) can explain the behavior of the recirculation zone at a macroscopic scale, 
notably the evolution of the recirculation length as a function of $S_{d}$. To achieve both objectives, four cases corresponding to the two macroscopic regimes (shallow and deep) were selected for a detailed analysis.

The paper is organized as follow. A first section details the experimental facility. A second section is devoted to select the four configurations corresponding to different macroscopic behaviors of the recirculation zone and to different mean flow properties. The connection with the local phenomena is enabled by the third section which details the properties of the mixing layers and compares them to more classical mixing layers. As these properties are linked to the $2 \mathrm{D}$ turbulent eddies behavior, the latter are studied in a fourth section. From all these results, the discussion sums-up the new knowledge on both objectives of the present paper: (i) the combined effect of the shallowness and of the lateral wall on the mixing layer and (ii) the connection of the (integral) recirculation behavior with the local mixing layer properties. Note that for the sake of clarity when comparing the present SSE mixing layers with more simple configurations from the literature, the following terminology is used: "deep" configurations refer to configurations with negligible vertical confinement, "unbounded" configurations refer to configurations with negligible lateral confinement and finally "free" mixing layers are both deep and unbounded.

\section{EXPERIMENTS}

\section{Experimental facility}

The experiments are conducted in an open-channel flume located in the Laboratory of Fluid Mechanics and Acoustics (LMFA) at the University of Lyon (INSA-Lyon, France) sketched in Fig.1. The flume is $L_{t}=8 \mathrm{~m}$ long, straight, with a constant streamwise slope of $0.18 \%$ and a symmetrical rectangular cross-section of width $B=0.8 \mathrm{~m}$. The flume is PVC made with a typical roughness height $\varepsilon \sim 5 \times 10^{-5} \mathrm{~m}$. A rectangular shape impervious block of width $d=0.2 \mathrm{~m}$ is included along the upstream part of the right bank over a length $L_{b}=3.56 \mathrm{~m}$. The expansion ratio thus equals $R_{b}=(B-d) / B=0.75$. The axis system is set as depicted on Fig.1 with the center located at the expansion section along the side wall, $x$ the streamwise axis and $y$ the crosswise axis. The discharge $Q$ is measured in the pumping loop using one of the two available electromagnetic 
flowmeters (Endress-Hauser): the first one in the range $Q=5-40 \mathrm{~L} / \mathrm{s}$ with an uncertainty $0.2 \mathrm{~L} / \mathrm{s}$, the other for $Q=0-5 \mathrm{~L} / \mathrm{s}$ with an uncertainty of $0.025 \mathrm{~L} / \mathrm{s}$. The upstream boundary condition consists of a grid buffer and a honeycomb with small mesh $(0.5 \mathrm{~cm}$ alveolus $)$ in order to stabilize the inflow. Moreover, a float board made of extruded polystyrene lies on the free surface in order to suppress the potential free surface oscillations. The downstream boundary condition consists of an adjustable tailgate, preceded by a stilling basin, allowing to precisely adjust the downstream water depth.

The velocity field is measured using a Vectrino+ Nortek side-looking ADV (Acoustic Doppler Velocimeter) mounted on an automatic displacement and recording carriage connected to a PC computer through LabVIEW software. In shallow conditions, this device permits to access the two horizontal velocity components $u, v$ along the streamwise $(x)$ and transverse $(y)$ directions respectively, with $u=\bar{u}+u$ ' where the overline refers to time-averaged and prime to fluctuating velocity component. Hollow glass spheres $(50 \mu \mathrm{m})$ and micro-bubbles are added to the water in the upstream tank to improve the acoustic backscattering. Spikes included in the raw ADV data are removed using the now classical Phase-Space Thresholding Method developed by Goring and Nikora (2002). Time convergence of all terms is ensured with measurements at a frequency of 30 $\mathrm{Hz}$ during 180 seconds. Moreover, when estimating the velocity variances (for the estimation of the normal Reynolds stresses) noise is removed using the spectral method proposed by Voulgaris and Trowbridge (1998). The measurement grid is composed of about one thousand points in the region downstream from the expansion $(0<x<1.3 L$ with $L$ the recirculation length and $0<y<3.5 d)$ with a spatial resolution of $2 \times 4 \mathrm{~cm}$ in the recirculation zone $(y<1.5 d)$ and $4 \times 4 \mathrm{~cm}$ in the free stream $(y>1.5 d)$, as shown in Fig.3. The velocity field is measured at an elevation equal to $0.4 h_{0}$ with $h_{0}$ and $U_{0}$ the water depth and mean streamwise velocity measured at the expansion section $(x=0)$ at a transverse distance of $y=1.5 d$. Finally, the water depth is measured using an ultrasonic probe (Baumer electric, uncertainty $0.15 \mathrm{~mm}$ ) also located on the automatic displacement carriage.

\section{FLOW DESCRIPTION}




\section{Selection of the experimental configurations}

Fig. 2 plots a series of 16 non-dimensional recirculation lengths configurations with increasing $S_{d}$ values presented by Chatelain et al. (2014). The recirculation length $L$ is defined as the distance from the expansion where the mean streamwise velocity along the side wall changes sign from negative (towards upstream) in the recirculation to positive (towards downstream) further downstream. The specific "bell" shape presented by Chatelain et al. (2014), which differs from the fitting curves proposed by Chu et al. (2004), exhibits a maximum non-dimensional length $L / d$ obtained for $S_{d} \sim 0.01$ and decreasing lengths for lower and higher $S_{d}$ values. Four configurations (F1 to F4) are selected here. Two configurations, F1 and F2, are specifically selected for the detailed analysis of the mixing layer characteristics. They are located on both sides of the "bell" with comparable recirculation lengths: $\mathrm{F} 1$ is a deep configuration $\left(S_{d}<0.01\right)$ and $\mathrm{F} 2$ a shallow configuration $\left(S_{d}>0.01\right)$. Two additional configurations are also considered for comparison: F3 located on the "bell" top and refered as a transitional flow and F4 an additional shallow configuration obtained with a larger bottom roughness (by addition of aluminum tear plates). All flow characteristics are detailed in Table 1.

\section{Mean flow properties}

As a first step, the full development of the flow in the upstream channel $(x<0)$ is verified (not shown here) so that the water depth, mean horizontal velocity components and Reynolds stresses along the center line of the incoming channel do not evolve when approaching the expansion. The mean velocity fields measured downstream from the expansion at $z=0.4 h_{0}$ elevation are plotted in Fig.3. The flow separates at $x / L=0$ and $y / d=1$, with a velocity vector almost parallel to $x$ axis and reattaches at $x / L=1$ where the mean streamwise velocity close to the wall changes sign from negative to positive. Note however that the reattachment point is known to vary in time due to the passing of coherent structure (e.g. Riviere et al. (2011)). A main recirculation cell forms for $y / d<1$ and $x / L<1$ and a small secondary cell near the corner at $x=y=0$ is also present (Chu et al. (2004)); as could be seen on a zoom of Fig.3 (not shown here). In the outer region $(y / d>1.5)$, the velocity field is deflected towards the recirculation region and decelerates along $x$ axis. 
In addition, the streamwise mean velocity normalized by $U_{0}$ is plotted along several crosssections in Fig.4 along with the separating streamline. First, it appears that the two separating streamlines differ as the shallow mixing layer (F2) approaches the side wall more rapidly than the deep one (F1). Moreover, while the velocity profiles for both configurations are very similar to each other within the recirculation zone and resemble measurements by Talstra (2011) (see their figure 3.11), they strongly differ in the outer region. For the shallow F2 configuration, the velocity profiles rapidly increase across the separating streamline and become almost uniform for $y / d>1.1$. Oppositely, for the deep F1 configuration, the mean streamwise velocity increases more gently along $y$ axis until $y / d>1.5$ and no uniformity is observed in the plotted region. This difference of behavior is in agreement with data from Babarutsi et al. (1989) when comparing their deep (in their figures 3 and 4) and shallow (figures 6 and 7) cases. The mean streamwise velocity profiles then appear to strongly differ from those self-similar reported for unbounded mixing layers (Bell and Mehta (1990)) and appear to be highly impacted by the lateral confinement and the shallowness.

Fig. 5 then plots along the same sections the dimensionless transverse gradient of streamwise mean velocity. This figure reveals that the maximum gradient is located along the separating streamline for $x / L<0.8-0.9$ and that further downstream it remains far from the side wall while the separating streamline reattaches. Moreover, the maximum gradient is measured at the expansion section and it decreases towards the reattachment point. The region of high gradient then spreads laterally when advancing towards downstream. The shape of velocity gradient herein is in agreement with all mixing layer configurations mentioned in the introduction section and thus confirms the existence of a mixing-layer along the separating streamline. Nevertheless, strong differences can be observed between the two flow configurations considered herein: the maximum gradient is stronger for the shallow case (F2) while the transverse extension of high gradient is higher for F1.

\section{Reynolds stress tensor}

The components of the 2D Reynolds stress tensor $\overline{u \prime^{2}}, \overline{v^{2}}$ and $-\overline{u / v^{\prime}}$ are plotted in dimensionless form in Fig. 6: the distributions are quite similar for the 3 terms. First, the stresses are 
maximum along the separating streamline (for $y / d<0.8-0.9$ ) and vanish on both sides (in the recirculation zone and the outer main flow). Moreover, the stress magnitudes are increasing in the upstream region of the mixing layer, reach a maximum at $x / L \sim 0.3$ to 0.5 and decrease downstream. These behaviors are in agreement with data from the SSE in the literature (Babarutsi et al. (1989) and Talstra (2011)) and with deep or shallow-unbounded mixing layers. Finally, when approaching the reattachment point, the maximum stresses are measured away from the side wall (near $y / d \sim 0.3$ to 0.5 ).

In order to compare the magnitude of the Reynolds stresses between both F1 and F2 configurations and with data from the literature, Tab.2 summarizes some available maximum Reynolds stresses magnitudes selected in a free and in a shallow unbounded mixing layers, in a backward facing step and in available shallow sudden expansions. For all configurations, the maximum streamwise normal stress exceeds both the crosswise normal stress and the shear stress: the crosswise normal stress equals about $40-50 \%$ and the shear stress about $35 \%$ of the streamwise normal stress except for some measurements from Talstra (2011) which are slightly out of these ranges. Moreover, for the sudden expansions (from Babarutsi et al. (1989) and present configurations), the maximum stress values decrease as $S_{d}$ increases. Globally, the Reynolds stress magnitudes appear to behave similarly as for deep and for unbounded mixing layers.

Fig. 7 plots the location of maximum velocity gradient and Reynolds stresses at each measured section along with the separating streamline. It confirms that the centerline of the mixing layer, defined here as the location of maximum velocity gradient, is also the location of maximum stresses and of the separating streamline as long as it remains far from the side wall $(x / L<0.8)$, i.e. as long as the effect of the lateral confinement is negligible. The mixing layer centerline and separating streamline then separate at $x / L \approx 0.8$ where the centerline remains apart from the wall (as previously reported for backward facing steps, see Chandrsuda and Bradshaw (1981)), at a distance $y / d=0.6$ for $\mathrm{F} 1$ and $y / d=0.3$, shorter, for $\mathrm{F} 2$. This result is a first hint that the shallowness affects the lateral confinement of the mixing layer: this difference of distance to the lateral wall at the reattachment section is connected to the wider mixing layer for F1, due to a weaker vertical 
confinement, as studied in next section.

\section{MIXING-LAYER CHARACTERISTICS}

\section{Definitions}

The width of the mixing layer is defined as the maximum slope thickness:

$$
\delta(x)=\frac{\Delta U(x)}{\left|\frac{\partial \bar{u}(x)}{\partial y}\right|_{\max }}
$$

This definition is commonly used in the literature regarding shallow mixing layers (e.g. Chu and Babarutsi (1988); Uijttewaal and Booij (2000); Van Prooijen and Uijttewaal (2002); Talstra (2011); Constantinescu (2013)) and, unlike for other definitions, does not require velocity plateaus on both sides of the mixing layer. This definition requires however the outer velocity difference $\Delta U(x)=U_{1}(x)-U_{2}(x)$ where $U_{1}(x)$ and $U_{2}(x)$ are the outer velocity magnitudes, respectively in the main flow and the recirculation zone.

The streamwise growth of the mixing-layer width is classically defined as a function of the ratio between the outer velocity difference and the center velocity $U_{c}(x)=\left(U_{1}(x)+U_{2}(x)\right) / 2$, reading:

$$
\frac{d \delta}{d x}=\alpha \frac{\Delta U(x)}{U_{c}(x)}
$$

where $\alpha$ is the spreading rate coefficient ; for free mixing layers, $\alpha=0.06-0.11$ (Pope (2008)) or $\alpha=0.085$ (Lesieur (1997)).

Finally, the mixing layer width is connected to the local bed friction number $S(x)$ (Chu et al. (1983)) as (using Eq.2)

$$
S(x)=\frac{c_{f} U_{c}(x)}{2 h\left|\frac{\partial \bar{u}(x)}{\partial y}\right|_{\max }}=\frac{c_{f} U_{c}(x)}{2 h \Delta U(x)} \delta(x)
$$

\section{Reported streamwise evolution of the mixing layer width from the literature}

Eq.3 reveals that the streamwise evolution of the mixing layer width $\delta(x)$ can be derived from the streamwise evolution of the outer velocity difference $\Delta U(x)$ and the center velocity $U_{c}(x)$, these evolutions being strongly affected by the characteristics of the mixing layer. 
A first case corresponds to the unbounded shallow mixing layer. Under specific assumptions, the depth-averaged momentum equations lead to $\Delta U(x)$ decreasing exponentially with the streamwise distance $x$ (Chu and Babarutsi (1988) ; Van Prooijen and Uijttewaal (2002)). Van Prooijen and Uijttewaal (2002) consider additionally a constant value of $U_{c}(x)$ and then obtain an exponential decay of the mixing layer width $\delta(x)$. This tendency confirms the stabilization of the mixing layer width observed in the literature on unbounded shallow mixing layers and is in satisfactorily agreement with their experimental data.

A second case corresponds to the field experiments of Sukhodolov et al. (2010) in a straight shallow river reach with an initial crosswise velocity gradient. The authors report a linear decrease of the outer velocity difference $\Delta U(x)$. Considering a constant value of the center velocity $U_{c}$, as for authors cited for the first case, leads to a parabolic evolution of the mixing layer width $\delta(x)$. These authors consider that the agreement is satisfactory with their field measurements using a fixed spreading rate coefficient $\alpha=0.11$. Note that Booij and Tukker (2001) obtained alternatively an exponential decay (see their cases A and D) and a linear decay (see their cases B and C) of the outer velocity difference $\Delta U(x)$.

A third case, the curved mixing layer observed in an open-channel confluence (Mignot et al. (2014b)) exhibits a linear decay of the outer velocity difference and a linear increase of the center velocity. The mixing layer width is thus described by a logarithmic law ; a reasonable agreement with experimental values is obtained, considering $\alpha=0.09$.

The present shallow sudden expansions then consist in a fourth case. Regarding the outer velocity difference, the faster outer velocity $U_{1}(x)$ in the free stream is measured at $y / d=1.5$ for each section while $U_{2}(x)$ is the outer velocity on the recirculation side. In their study around a square cylinder, Lyn and Rodi (1994) define $U_{2}$ as the minimum velocity (negative) measured within the recirculation region along each profile. In his sudden expansion, Talstra (2011) considers $U_{2}(x)=0$, i.e. the mean streamwise velocity in the zero-discharge recirculation region. This definition is used in the present paper so that the outer velocity difference reads $\Delta U(x)=U_{1}(x)-U_{2}(x)=U_{1}(x)$ and the center velocity reads $U_{c}(x)=\left(U_{1}(x)+U_{2}(x)\right) / 2=U_{1}(x) / 2$ so that the ratio $\frac{\Delta U(x)}{U_{c}(x)}=2$ is 
constant along the whole mixing layer. Hence, the longitudinal grows of the mixing layer in Eq.3 obeys to:

$$
\frac{d \delta}{d x}=\alpha \frac{\Delta U(x)}{U_{c}(x)}=2 \alpha
$$

Please note that Talstra (2011) considers a different definition: $U_{c}(x)=U_{1}(x=0) / 2 \approx U_{0} / 2$ and thus does not end up with the same expression as Eq.5.

\section{Present streamwise evolution of the mixing layer width}

The streamwise evolution of the measured mixing layer width $\delta$ is plotted in Fig. 8. For the four cases, the flow development upstream from the expansion leads to similar mixing layer widths at the expansion, $\delta_{0} / d=\delta(x=0) / d \approx 0.12$. However, further downstream, the mixing layer expands differently for the different cases. For the deep configuration F1, $\delta$ increases linearly (as predicted by Eq.5) with an increasing rate up to $x / L=0.7$ corresponding to $\alpha=0.07$. Further downstream, the linear spreading rate suddenly reduces, as demonstrated by the linear regressions on Fig. 8. For the shallow cases F2, F3 and F4, $\delta$ also increases linearly up to $x / L=0.7$ (as predicted by Eq.5), with rates smaller than for F1 and which minimum is obtained for the shallower cases (F2 and F4): $\alpha \approx 0.06$. Again, the spreading rate suddenly reduces at $x / L=0.5$ to 0.7 , but much more than F1 as the curves experience a plateau: $\delta(x)$ becomes constant.

The sudden decrease of this growth rate cannot be attributed to the bed friction. Indeed, as in our case $\frac{\Delta U(x)}{U_{c}(x)}=2$, the local bed friction number from Eq.4 becomes $S(x)=\delta(x) c_{f} / 4 h$. Moreover, assuming that both the friction coefficient and the water depth are almost constant along the mixing layer, as for Babarutsi et al. (1989), see Eq.1, the final expression used herein reads:

$$
S(x)=\frac{\delta(x) c_{f 0}}{4 h_{0}}
$$

The abrupt change of spreading rate occurs for $S(x)=0.012$ for case $\mathrm{F} 1$ and the plateaus $(\alpha \approx 0)$ occur for $S(x)=0.003-0.025$ (referred to as $S_{\text {max }}$ in Tab.1) for F2, F3, F4 (see Fig. 8). All these values are significantly smaller than the critical values proposed in the literature $\left(S_{c}=\mathrm{O}(0.1)\right.$, see 
the introduction section). Hence, no damping of the size of the coherent structures (corresponding whether to the dominant or the most unstable mode, see introduction) due to bed friction is expected.

The rupture in the spreading rate of the mixing layer width, is rather attributed to the interaction of the mixing layer with the side wall $(y=0)$, i.e. to the lateral confinement, itself being influenced by the shallowness, as discussed above (see Fig. 7). In Fig.9, the streamwise evolution of the centerline of the mixing layer ( $y_{c}$, the location of maximum velocity gradient) is plotted along with the half width $\delta(x) / 2$ extension on both sides, assuming a symmetrical extension distribution. It shows that, both for shallow and deep cases, the downstream end of the recirculation corresponds to the intersection of the mixing layer boundary with the side wall: $\left(y_{c}-\delta / 2\right) \sim 0$ at $x / L=1$. And as, at the reattachment point, $\delta$ is much larger for deep than for shallow cases, the centerline of the mixing layer ends up further from the wall. Note that Biancafiore et al. (2011) already revealed through DNS calculations for a laminar transversely-confined mixing layer that the increasing width of the mixing layer suddenly stops at a given streamwise axis due to the lateral confinement.

To conclude, the SSE mixing layers behave as free mixing layers in the upstream region $(x / L<=0.6-0.7)$, with a constant spreading rate, and as laterally-bounded mixing layers further downstream, with an abruptly decreasing spreading rate that differs for deep and shallow cases. This sudden decrease is thus governed by the combined effects of shallowness (which imposes 2D large turbulent eddies) and of the interaction of these eddies with the lateral wall - which itself depends on the shallowness.

\section{ROLE OF THE COHERENT STRUCTURES IN THE MIXING LAYER}

\section{Coherent structures in the mixing layer}

The aim of the present section is to estimate the peak frequency associated to the coherent structures advected along the mixing layer. Fig.10 plots the energy spectrum of the transverse velocity component $v$ in the mixing layer at $x / L=0.2$ and $y=y_{c}$ while Fig.11 plots similar spectra every $x=L / 13$ distance (at $y=y_{c}$ ), in a similar manner as Hertzberg and Ho (1995) or White and 
Nepf (2007) for other mixing layer configurations, with the peak indicated by a symbol for each spectrum.

For averaged frequencies $(f=0.7$ to $7 \mathrm{~Hz})$, the main inertial range characterized by the $-5 / 3$ cascade is observed. In the low frequency range, the peak frequency $f_{p}$ corresponding to the dominating vortex passing frequency appears to differ as a function of the shallowness: at $x / L=0.2$, $f_{p} \sim 0.6 \mathrm{~Hz}$ for the shallow $\mathrm{F} 2$ case, while $f_{p} \sim 0.3 \mathrm{~Hz}$ for the deep F1 case (see Fig.10). For F1, this peak frequency decreases towards downstream from $f_{p}=0.3 \mathrm{~Hz}$ to $f_{p}=0.1 \mathrm{~Hz}$ at $x / L=0.5$ and an even lower frequency at the reattachment section $(x / L=1)$. For F2, the peak frequency decreases less rapidly from $f_{p}=0.6 \mathrm{~Hz}$ to $f_{p}=0.4 \mathrm{~Hz}$ at $x / L=0.5$ and $f_{p}=0.2 \mathrm{~Hz}$ at $x / L=1$. The decreasing peak frequency behaviors along the streamwise axis are in agreement with observations from Talstra (2011) and Uijttewaal and Booij (2000). Moreover, the vortex passing frequency appears to decrease less rapidly for the shallow case than for the deep case and this is connected to the dynamic of large eddies (see below).

The eddies can be directly observed using time-exposure photographs of floating sawdust (see Riviere et al. (2011)). Photographs for the shallow case F2 in Fig.12 exhibit 2D vortices of vertical axis, with an increasing size during their advection. The behavior appears quite reproducible from one vortex to another. The reattachment location can be identified, and varies from one photograph to another around the $x / L=1$ location, depending on the position of the vortices. On Fig.13, the deep case F1 appears more complex: again, vortices of vertical axis are clearly visible, with bigger sizes, but with less reproducible trajectories and sizes. The flow reattachment cannot be located as easily as for the shallow case F2.

In order to obtain more quantitative vortex characteristics, the auto-correlation function of the transverse velocity fluctuation $R v v$ is computed along the centerline of the mixing layer $\left(y=y_{c}\right)$, at $x / L=0.2,0.5,0.8$ and 1 (see Fig.14). The characteristic time scale of the large scale coherent structures is considered as the time shift between two successive peaks, as defined by Constantinescu (2013). The signal is more complex for the deep case F1, confirming that eddies are less reproducible on the photographs, but the two cases exhibit similar trends (see Fig.14). Firstly, the 
time shift $\tau$ between two successive eddies increases as $x / L$ increases, indicating that the $2 \mathrm{D}$ eddies grow in the streamwise direction. Secondly, the coherence of the signal is worse for $x / L=0.2$ than further downstream, corresponding to the initial development of eddies. Thirdly, the time shift for the shallow case F2 is much shorter than for the deep condition F1, corroborating the difference of peak frequencies observed through the spectrum analysis (Figs. $10-11$ ).

Tab.3 compares the results from both techniques (data from the photographs and from the ADV, including the autocorrelation function) for two $x / L$ values, for the shallow case F2. Estimates of the transverse (along $y$ ) length scale of vortices from photographs compare well with the mixing layer width derived from the mean velocity fields. Estimates of the convection velocity by measuring the vortices center displacement between two successive photographs compare well with the measured local mean velocity (for the latter, two values are provided, measured on both sides of the maximum gradient location). Estimates of the vortex passing frequencies from photographs compare well with the spectra peak frequencies at the same location. Finally, estimates of the longitudinal length (along $x$ ) of vortices from photographs compare well with the integral length $\Lambda$ obtained by multiplying the local mean velocity with the characteristic times from the autocorrelation Rvv on both sides of the maximum gradient location (Uijttewaal and Booij (2000)). The same comparison is performed for the deep case F1 at $x / L=0.5$, in Tab.4. The agreement is slightly lower (due to the difficulty in estimating accurate values from the photographs) than for case F2 but the conclusions are alike: the specific tendency of the mixing layer when approaching the reattachment point, attributed to the width of the mixing layer can actually be attributed to the impact of the coherent vortices with the lateral wall.

To correlate further the role of vortices on the behavior of the mixing layer and on the flow reattachment, the dimensionless mixing layer width $\delta / d$ is plotted in Fig.15 as a function of $x / L$, along with the integral longitudinal length scale of vortices $\Lambda / d$. The behaviors of $\delta / d$ and of $\Lambda / d$ are similar: linear increase with a slope shortage for the deep case F1 and linear increase followed by a plateau for the shallow case F2. Considering the two different vertical axes, the mixing layer width is about 3.5 times smaller than the longitudinal integral length scale. This ratio corresponds 
to the longitudinal/transverse diameters ratio observed in the photographs (Fig.12 and Tab.3) and to the ratios obtained experimentally (Uijttewaal and Booij (2000)) or numerically (Cheng and Constantinescu (2014)) for an unbounded shallow mixing layer of larger scale. In his experimental study, Talstra (2011) reports a ratio of about 2 ; the only explanation for this discrepancy is that he used a spatial correlation based on the vector potential function, instead of a time autorrelation.

To summarize, vortices appear to increase in terms both of streamwise and transverse extension along the development of the mixing layer. For the case where the coherent structures are strongly 2D (F2), the streamwise evolution of their streamwise length is in particularly fair agreement with the streamwise evolution of the mixing layer width: a linear increase in the upstream region followed by a plateau when approaching the reattachment point, due to their interaction with the lateral wall. The deeper case F1 suffers from a huge scattering in the measurements of coherent structure streamwise length: this prevents from emitting so definite conclusions.

To conclude, the shallowness, accounted by the integral bed friction number $S_{d}$, influences the vortices size in the same way as for the mixing layer width (see above). In the upstream part of the mixing layer $(x / L<=0.6-0.7)$, both the mixing layer and the large scale vortices evolve similarly for $\mathrm{F} 1$ and $\mathrm{F} 2$. Further downstream $(x / L>0.6-0.7)$, both the mixing layer and the vortices stop growing for the shallow cases (F2, F3, F4) whereas they both go on growing - though more gently - for the deep case F1. Moreover, the behavior of the mixing layer when approaching the wall is a consequence of the interaction of the $2 \mathrm{D}$ large scale vortices with the lateral wall. This interaction is affected by the shallowness and could be linked to the weaker 2D character of the vortices in the deep case, as the water depth increases by a factor 7.4 from F2 to F1.

\section{DISCUSSION AND CONCLUSIONS}

This paper characterized experimentally the mixing layer that develops at the interface between the main flow and the recirculation zone created by a shallow sudden expansion (SSE). These mixing layers are confined vertically between the channel bed and the free-surface (shallowness) and bounded laterally by the wall (lateral confinement). The first objective was to identify the combined influence of the shallowness and of the lateral confinement on the SSE mixing layers, by 
checking their differences with simpler mixing layers available in the literature, i.e. either shallow and unbounded or deep and laterally confined mixing layers. The second objective was to check the link between the macroscopic behavior of the recirculation (mainly its length) and the local behavior of the mixing layer. Hence, four experimental configurations with identical geometry but varying shallowness were carefully selected to cover all regimes of behavior of the recirculation: F1 referred to as deep, F3 as transitional, F2 as shallow and F4 as shallow with a high bed friction number value.

The first conclusion is related to the role of the shallowness and of the lateral confinement on the mixing layer compared to simpler mixing layers. In its upstream region, the SSE mixing layer exhibits classical features of all mixing layers. It is the location of high velocity gradients and Reynolds stresses, both with a maximum magnitude at the centerline of the mixing layer and a rapidly decreasing magnitude on both sides. This centerline merges into the separating streamline originating from the upstream corner. The width of the mixing layer increases linearly towards downstream from an initial magnitude at the separation corner as for any free (deep and unbounded) mixing layer. In its downstream region, this behavior changes: the expansion of the mixing layer width stops for the shallow cases while it is reduced for the deep case. The damping of the coherent structures by the bed friction appears not to be responsible of this specific behavior, as the local bed friction numbers remain too small in our whole experimental range. The reason for this sudden reduction of width expansion is rather the interaction of the lateral wall with the coherent turbulent structures in the vicinity of the reattachment region. This interaction appears to differ for the shallow and the deep flows, maybe connected to weakening of the $2 \mathrm{D}$ character of coherent structures in the mixing layer when increasing the water depth.

The second conclusion is related to the connection of $S_{d}$ with $S(x)$. It was shown herein and in the literature that the global flow shallowness $\left(S_{d}\right)$ strongly influences the macroscopic behavior of the recirculation, in particular its dimensionless length $L / d$. It is then tempting to relate this macroscopic effect to the degree of damping of the coherent structures in the mixing layer. Nevertheless, as already mentioned above, a bed friction damping was not observed in the present 
mixing layers. No evident relationship can be obtained between the global flow shallowness and the local mixing layer shallowness. In other words, a critical value of the integral bed friction number $S_{d}$ (Eq. 1), separating the "deep water flow" and the "shallow water flow" behaviors in Fig. 2, cannot be related to a critical value of the local bed friction number $S(x)$ (Eq. 6), indicating the appearance of a noticeable damping of the coherent structures by the bed friction. For all that, the global flow shallowness $\left(S_{d}\right)$ jointly affects the recirculation length and the coherent structures evolution and size. Future work, using different techniques such as PIV, should be devoted to characterize these coherent structure dynamics more in details all along their advection within the mixing layer, notably in the vicinity of the reattachment point so that where their exact interaction with the side wall could be better understood, for different values of the global flow shallowness. It would then be possible to definitely establish if the large scale vortices play a role in the huge variation of the recirculation length as a function of the shallowness downstream expansions.

\section{REFERENCES}

Alavian, V. and Chu, V. (1985). "Turbulent exchange flow in shallow compound channel." Proc., 21 st Congress of IAHR Melbourne, (CD-ROM), Melbourne, Australia, 446-451.

Babarutsi, S., Ganoulis, J., and Chu, V. (1989). "Experimental investigation of shallow recirculating flows." J. Hydraul. Eng. 115(7), 906-924.

Bell, J. and Mehta, R. (1990). "Development of a two-stream mixing layer from tripped and untripped boundary layers." AIAA J. 28(12), 2034-2042.

Biancafiore, L., Gallaire, F., and Pasquetti, R. (2011). "Influence of confinement on a twodimensional wake.” J. Fluid. Mech. 688, 297-320.

Booij, R. and Tukker, J. (2001). "Integral model of shallow mixing layers.” J. Hydraulic Research 39(2), 169-179.

Chandrsuda, C. and Bradshaw, P. (1981). “Turbulence structure of a reattaching mixing layer.” J. Fluid. Mech. 110, 171-194.

Chatelain, M., Han, L., Riviere, N., and Mignot, E. (2014). "Physics of shallow recirculation zones 
downstream lateral expansions." Proc., Int. Conference on Fluvial Hydraulics - River Flow 2014, (CD-ROM), Lausanne, Switzerland, 701-709.

Cheng, Z. and Constantinescu, G. (2014). "Spatial development of a constant-depth shallow mixing layer in a long channel." Proc., Int. Conference on Fluvial Hydraulics - River Flow 2014, (CD-ROM), Lausanne, Switzerland, 155-164.

Chu, V. and Babarutsi, S. (1988). "Confinement and bed friction effects in shallow turbulent mixing layers." J. Hydraul. Eng. 114, 1257-1274.

Chu, V., Liu, F., and Altai, W. (2004). "Friction and confinement effects on a shallow recirculating flow." Journal of Environmental and Engineering Science 3(5),463-475.

Chu, V., Wu, J., and Khayat, R. (1983). "Stability of turbulent shear flows in shallow channels." Proc. of the 20th Congress of IAHR, (CD-ROM), Moscow, Russia, 128-133.

Chu, V., Wu, J., and Khayat, R. (1991). "Stability of transverse shear flows in shallow open channels." J. Hydraul. Eng. 117(10), 1370-1388.

Constantinescu, G. (2013). "Les of shallow mixing interfaces :a review..” Envir. Fluid Mech. 14(5), 971-996.

Engelhardt, C., Kruger, A., Sukhodolov, A., and Nicklisch, A. (2004). "A study of phytoplankton spatial distributions, flow structure and characteristics of mixing in a river reach with groynes." Journal of Plankton Research, 26(11), 1351-1366.

Goring, G. and Nikora, V. (2002). "Despiking acoustic doppler velocimeter data.” J. Hydraul. Eng. 128(1), 117-126.

Hertzberg, J. and Ho, C. (1995). "Three-dimensional vortex dynamics in a rectangular sudden expansion.” J. Fluid Mech. 289, 1-27.

Jovic, S. and Driver, D. (1994). "Backward-facing step measurement at low reynolds number, reh=5000.” NASA Tech. Mem. 108807.

Kasagi, N. and Matsunaga, A. (1995). "Three-dimensional particle-tracking velocimetry measurement of turbulent statistics and energy budget in a backward facing step flow." International Journal of Heat and Fluid flow 16, 477-485. 
Lesieur, M. (1997). Turbulence in Fluids. Kluwer Academic, Norwell, U.S.A.

Li, X. and Djilali, N. (1995). "On the scaling of separation bubbles.” JSME Int. J. Series B 38(4), $541-548$.

Loucks, R. and Wallace, J. (2012). "Velocity and velocity gradient based properties of a turbulent plane mixing layer." Journal of Fluid Mechanics, 699, 280-319.

Lyn, D. and Rodi, W. (1994). "The flapping shear layer formed by flow separation from the forward corner of a square cylinder.” J. Fluid Mech. 267, 353-376.

Mignot, E., Doppler, D., Riviere, N., Vinkovic, I., Gence, J., and Simoens, S. (2014a). "Double averaging analysis and local flow characterization of near bed turbulence in gravel-bed channel flows.” J. Hydraul. Eng. 140(3), 280-290.

Mignot, E., Vinkovic, I., Doppler, D., and Riviere, N. (2014b). "Mixing layer in open-channel junction flows." Envir. Fluid Mech. 14(5), 1027-1041.

O’Connor, B., Hondzo, M., and Harvey, J. (2010). "Predictive modeling of transient storage and nutrient uptake: implications for stream restoration." Journal of Hydraulic Engineering, 136(12).

Pope, S. (2008). Turbulence flows. Cambridge University Press, Cambridge, United Kingdom.

Riviere, N., Badin, B., Bomchil, Y., and Proust, S. (2008). "Recirculation zones downstream open channel expansions." 4th Edition of the International Conference on Fluvial Hydraulics River Flow 2008, Izmir, Turkey, 2233-2238.

Riviere, N., Gautier, S., and Mignot, E. (2011). "Experimental characterization of flow reattachment downstream open channel expansions." Proceedings of the 34th World Congress of the International Association for Hydro- Environment Research and Engineering, Barton, Australia, $3745-3752$.

Sukhodolov, A., Schnauder, I., and Uijttewaal, W. (2010). "Dynamics of shallow lateral shear layers: experimental study in a river with a sandy bed." Water Resour. Res. 46, W11519.

Talstra, H. (2011). "Large-scale turbulence structures in shallow separating flows." Ph.D. thesis, Technische Universiteit Delft, Delft, Netherlands, 
$<$ http://repository.tudelft.nl/view/ir/uuid\%3A922e297b-19b9-4399-808d-23e837401a52/>.

Uijttewaal, W. and Booij, R. (2000). "Effects of shallowness on the development of free-surface mixing layers." Phys. Fluids 12(2), 392-402.

Van Prooijen, B. and Uijttewaal, W. (2002). "A linear approach for the evolution of coherent structures in shallow mixing layers." Phys. Fluids 4(12), 4105-4114.

Voulgaris, G. and Trowbridge, J. (1998). "Evaluation of the acoustic doppler velocimeter (adv) for turbulence measurements.” J. Atmos. Oceanic Technol. 15, 272-289.

White, B. and Nepf, H. (2007). "Shear instability and coherent structures in shallow flow adjacent to a porous layer." J. Fluid Mech. 593, 1-32.

Wygnanski, I. and Fiedler, H. (1970). "The two-dimensional mixing region.” J. Fluid Mech. 41(2), $327-361$. 


$$
\begin{aligned}
S & =\text { bed friction number; } \\
S_{d} & =\text { global bed friction number; } \\
S_{c} & =\text { critical bed friction number; } \\
c_{f} & =\text { friction coefficient; } \\
d & =\text { expansion width }(\mathrm{m}) ; \\
h & =\text { water depth }(\mathrm{m}) ; \\
B & =\text { total width of channel }(\mathrm{m}) ; \\
R_{b} & =\text { expansion ratio }=(B-d) / B ; \\
u & =\text { streamwise velocity }(\mathrm{m} / \mathrm{s}) ; \\
v & =\text { transverse velocity }(\mathrm{m} / \mathrm{s}) ;
\end{aligned}
$$

$$
\begin{aligned}
U_{1} \text { and } U_{2} & =\text { outer velocities }(\mathrm{m} / \mathrm{s}) \\
U_{c} & =\text { mean center velocity }(\mathrm{m} / \mathrm{s}) \\
\Delta U & =\text { outer velocity difference }(\mathrm{m} / \mathrm{s}) \\
f_{p} & =\text { peak frequency corresponding to the dominating vortex }(\mathrm{Hz}) \\
Q & =\text { inlet discharge }(1 / \mathrm{s}) \\
L & =\text { length of the recirculation zone }(\mathrm{m}) \\
L_{b} & =\text { length of the upstream flow region }(\mathrm{m}) \\
L_{t} & =\text { total channel length }(\mathrm{m}) ; \\
\delta & =\text { width of the mixing layer }(\mathrm{m}) \\
\alpha & =\text { grow rate of the mixing layer width; } \\
\Lambda & =\text { integral length scale }(\mathrm{m}) ;
\end{aligned}
$$




\section{List of Tables}

1 Flow characteristics for all studied configurations . . . . . . . . . . . . 27

2 The maximum measured Reynolds stresses compared with literature data (with $\Delta U_{0}$ the outer velocity difference at the upstream section where the mixing layer initiates). Note: FML means free mixing layer (White and Nepf (2007)), BFS means backward facing step (Chandrsuda and Bradshaw (1981)), SUM means shallow unbounded mixing layer (Uijttewaal and Booij (2000)) and SSE means shallow sudden expansion with SSE1 from Babarutsi et al. (1989), SSE2 from Talstra (2011) and SSE3 are present data. . . . . . . . . . . . . . . . 28

3 Characteristics of vortices and of the mixing layer for case F2 at two locations . . 29

4 The characteristics of the vortices passing along the mixing layer for F1 . . . . . 30 
TABLE 1. Flow characteristics for all studied configurations

\begin{tabular}{lcccccccccc}
\hline Test & $Q(1 / \mathrm{s})$ & $U_{0}(\mathrm{~m} / \mathrm{s})$ & $h_{0}(\mathrm{~m})$ & $c_{f 0}$ & $S_{d}$ & $L(\mathrm{~m})$ & $L / d$ & $S_{\max }$ & $\mathrm{Fr}_{0}$ & $\mathrm{Re}_{0}$ \\
\hline F1 (deep) & 20 & 0.23 & 0.156 & 0.0049 & 0.0032 & 1.29 & 6.45 & - & 0.19 & $9.4 \times 10^{4}$ \\
F2 (shallow) & 4.05 & 0.36 & 0.021 & 0.0068 & 0.032 & 1.16 & 5.80 & 0.009 & 0.79 & $2.8 \times 10^{4}$ \\
F3 (transitional) & 16.15 & 0.55 & 0.050 & 0.0050 & 0.01 & 1.99 & 9.95 & 0.003 & 0.79 & $9.4 \times 10^{4}$ \\
F4 (shallow) & 2.363 & 0.20 & 0.022 & 0.0195 & 0.089 & 0.86 & 4.3 & 0.025 & 0.43 & $1.6 \times 10^{4}$ \\
\hline
\end{tabular}


TABLE 2. The maximum measured Reynolds stresses compared with literature data (with $\Delta U_{0}$ the outer velocity difference at the upstream section where the mixing layer initiates). Note: FML means free mixing layer (White and Nepf (2007)), BFS means backward facing step (Chandrsuda and Bradshaw (1981)), SUM means shallow unbounded mixing layer (Uijttewaal and Booij (2000)) and SSE means shallow sudden expansion with SSE1 from Babarutsi et al. (1989), SSE2 from Talstra (2011) and SSE3 are present data.

\begin{tabular}{llccccccccc}
\hline & FML & BFS & SUM & \multicolumn{2}{c}{ SSE1 } & \multicolumn{3}{c}{ SSE2 } & \multicolumn{2}{c}{ SSE3 } \\
& & & & & & Case 1 & Case 2 & Case 3 & F1 & F2 \\
$S_{d}$ & & & & 0.0098 & 0.098 & 0.022 & 0.015 & 0.007 & 0.0032 & 0.032 \\
\hline$\overline{u^{2}} / \Delta U_{0}^{2}(\%)$ & 3.1 & 2.7 & 2.97 & 5.4 & 2.25 & 0.5 & 0.9 & 1.2 & 2.38 & 1.2 \\
$\overline{v \prime^{2}} / \Delta U_{0}^{2}(\%)$ & 1.9 & 1.5 & 1.36 & - & - & 0.3 & 0.7 & 0.4 & 0.88 & 0.6 \\
$-\overline{u / v \prime} / \Delta U_{0}^{2}(\%)$ & 0.925 & 1 & 1.04 & - & - & 0.2 & 0.4 & 0.2 & 0.74 & 0.5 \\
$\overline{v \prime^{2}} / \overline{u \prime^{2}}(\%)$ & 61 & 56 & 46 & - & - & 80 & 78 & 25 & 37 & 50 \\
$-\overline{u / v \prime} / \overline{u \prime^{2}}(\%)$ & 30 & 37 & 35 & - & - & 40 & 44 & 17 & 31 & 42 \\
\hline
\end{tabular}


TABLE 3. Characteristics of vortices and of the mixing layer for case $F 2$ at two locations

\begin{tabular}{lcccc}
\hline & \multicolumn{2}{c}{$x / L \sim 0.4$} & \multicolumn{2}{c}{$x / L \sim 0.7$} \\
& Photograph & ADV & Photograph & ADV \\
\hline Transverse size $(\mathrm{m})$ & 0.09 & $\delta=0.07$ & 0.12 & $\delta=0.11$ \\
$U_{\text {advection }}(\mathrm{m} / \mathrm{s})$ & 0.25 & $0.14-0.24$ & 0.2 & $0.13-0.2$ \\
Frequency $(\mathrm{Hz})$ & 0.59 & 0.6 & 0.44 & 0.47 \\
Streamwise size $(\mathrm{m})$ & 0.3 & $\Lambda=0.24-0.36$ & 0.36 & $\Lambda=0.31-0.42$ \\
\hline
\end{tabular}


TABLE 4. The characteristics of the vortices passing along the mixing layer for F1

\begin{tabular}{lcc}
\hline & \multicolumn{2}{c}{$x / L \sim 0.5$} \\
& Photograph & ADV \\
\hline Thickness $(\mathrm{m})$ & 0.2 & $\delta=0.13$ \\
$U_{\text {advection }}(\mathrm{m} / \mathrm{s})$ & 0.06 & $0.08-0.12$ \\
Frequency $(\mathrm{Hz})$ & 0.13 & 0.18 \\
Streamwise size $(\mathrm{m})$ & $0.25-0.44$ & $\Lambda=0.35-0.61$ \\
\hline
\end{tabular}




\section{List of Figures}

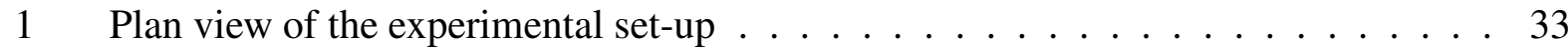

2 Evolution of the non-dimensional recirculation length as a function of the bed friction number with the selected configurations . . . . . . . . . . . . . . 34

3 Time-averaged velocity field along with selected streamlines (plain lines-), the bottom plot is a zoom of the top plot in the recirculation zone where $y / d<1 \ldots 35$

4 Evolution of transverse profiles of mean streamwise velocity along a few selected sections ( $\circ$ refers to F1 and $\boldsymbol{\Delta}$ refers to F2) with the separating streamlines plotted

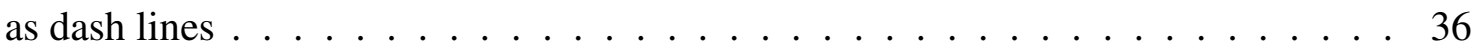

5 Evolution of the transverse gradient of mean streamwise velocity with the separating streamlines plotted as dash lines . . . . . . . . . . . . . . . 37

6 Evolution of the Reynolds stress terms with the separating streamlines plotted as dash lines. Note: the different scales for the streamwise normal term and both

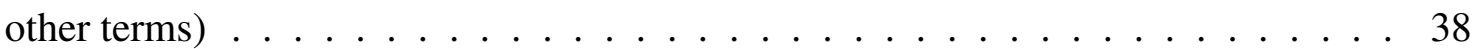

7 Location of maximum velocity gradient $(\triangleleft), \overline{v \prime^{2}}(\circ), \overline{v^{2}}(+)$ and $-\overline{u v^{\prime}}(\diamond)$ along with the separating streamline plotted as dash lines $\ldots$. . . . . . . . . . . . 39

8 Streamwise evolution of the measured (symbol) and fitted (plain lines -) nondimensional mixing layer width $\ldots \ldots \ldots$. . . . . . . . . . . 40

9 Evolution of the mixing layer centerline (symbols) and the estimated mixing layer boundaries $($ dash lines --$) \ldots \ldots \ldots \ldots \ldots$. . . . . . . . . . . . . . .

10 Energy spectrum of the transverse velocity component $v$ along the centerline of the mixing layer at $x=0.2 L \ldots \ldots \ldots \ldots \ldots \ldots \ldots \ldots$

11 Streamwise evolution of the energy spectrum of transverse velocity along the mixing layer centerline from $x / L=0$ to $x / L=1$. Note: the symbols presents the peak value of each $x / L$ section.

12 Successive $0.5 \mathrm{~s}$ exposure photographs for case F2. Dotted line : marks the location of the instantaneous reattachment . . . . . . . . . . . . . . . . . . 44 
14 Auto-correlation function of the transverse velocity fluctuation $R v v$ in different section of $x / L$ along the centerline of the mixing layer $\ldots \ldots$. . . . . . . . 46

15 The comparison between the mixing layer width (definition of Eq.2, left axis with symbols $\circ$ and $\triangle$ ) and longitudinal length scale of vortices (derived from the autocorrelation, right axis with symbols $\bullet$ and $\mathbf{\Delta}$ ), along the mixing layer centerline from $x / L=0$ to $x / L=1 \ldots \ldots \ldots \ldots \ldots \ldots \ldots \ldots$ 


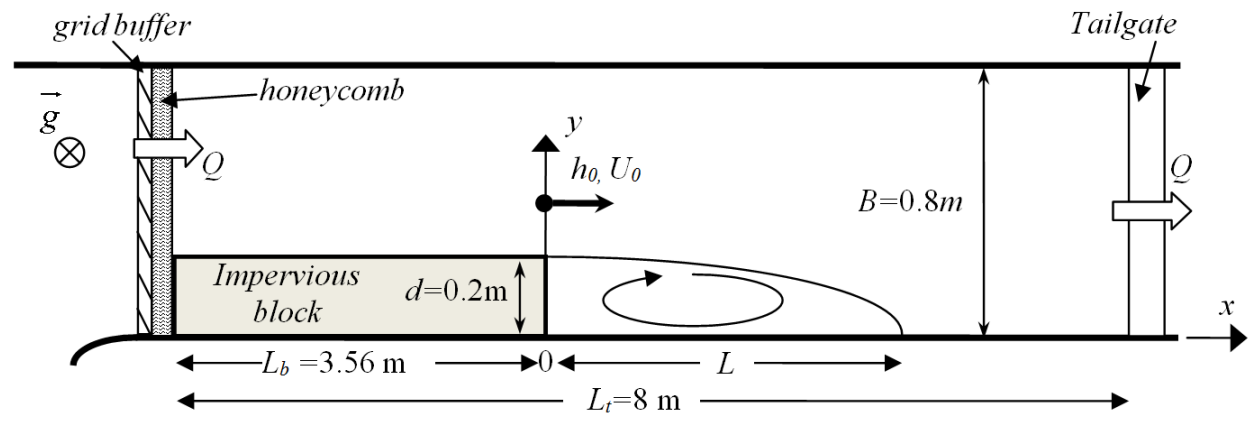

FIG. 1. Plan view of the experimental set-up 


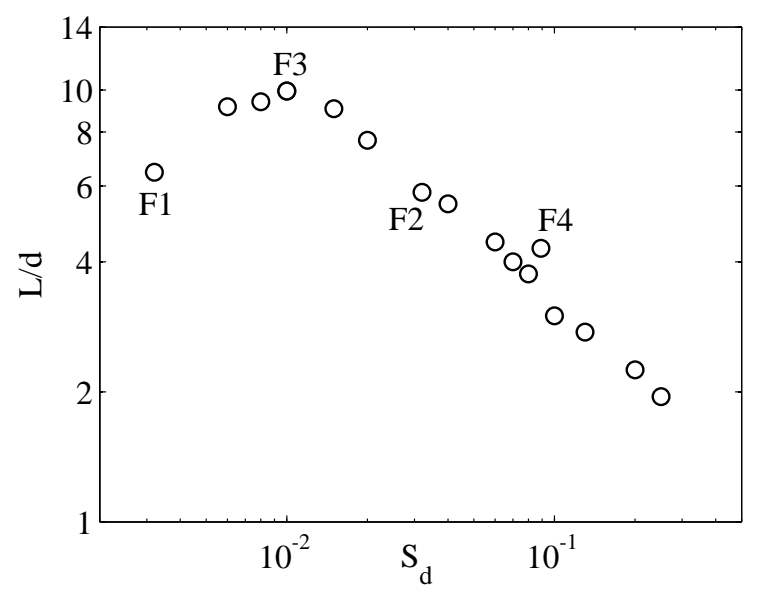

FIG. 2. Evolution of the non-dimensional recirculation length as a function of the bed friction number with the selected configurations 

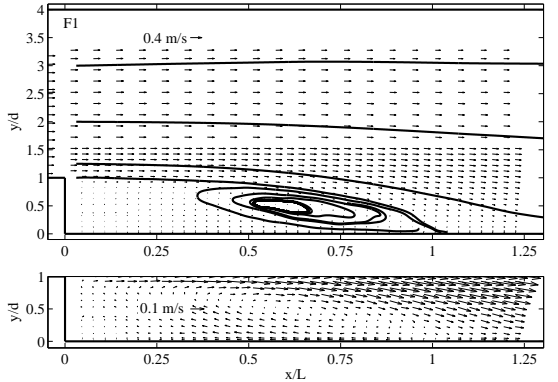
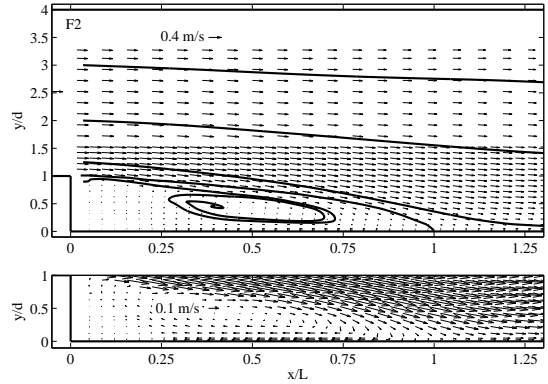

FIG. 3. Time-averaged velocity field along with selected streamlines (plain lines-), the bottom plot is a zoom of the top plot in the recirculation zone where $y / d<1$ 

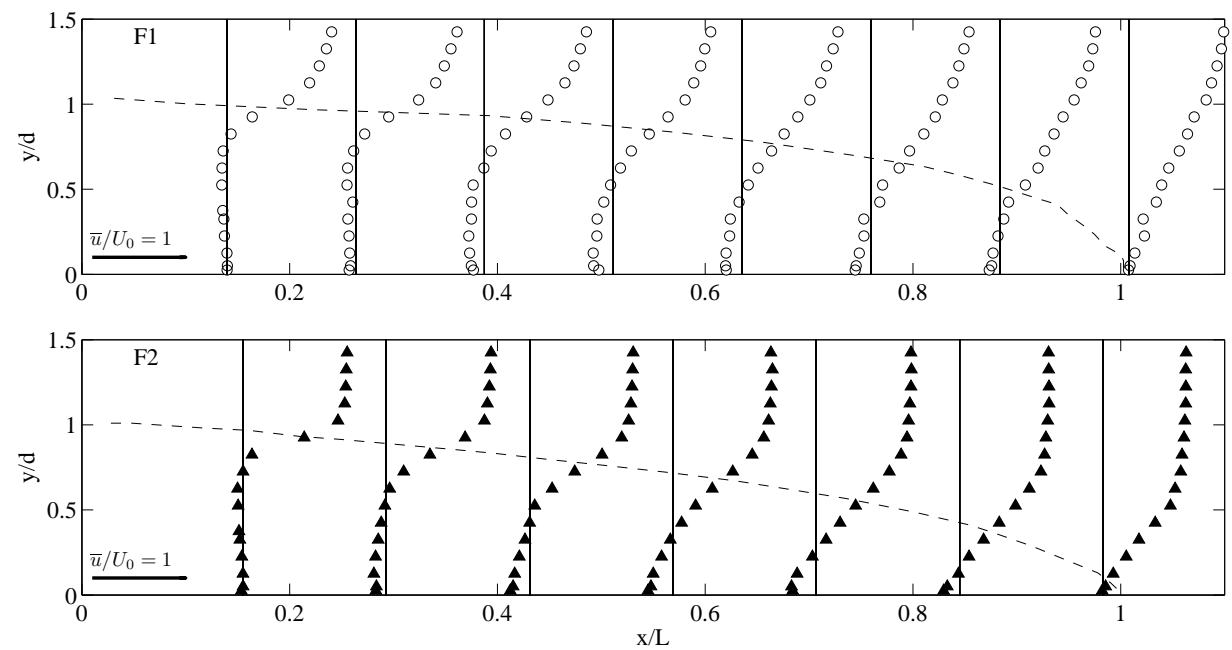

FIG. 4. Evolution of transverse profiles of mean streamwise velocity along a few selected sections ( $\circ$ refers to $F 1$ and $\Delta$ refers to $F 2$ ) with the separating streamlines plotted as dash lines 

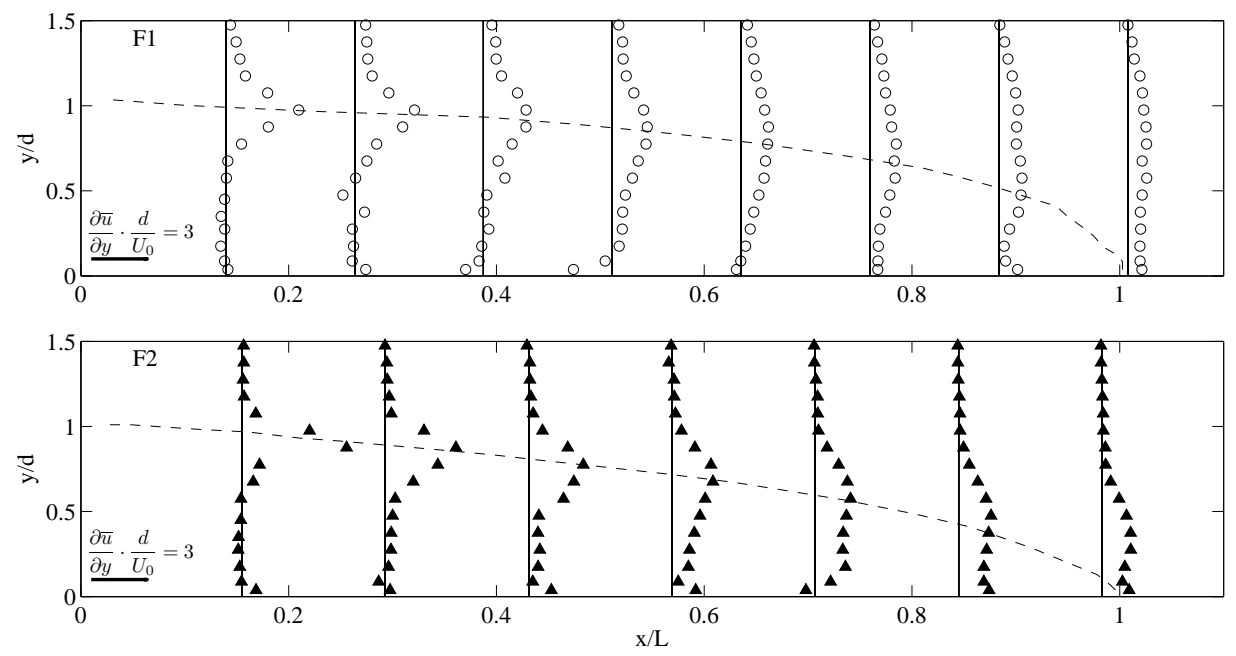

FIG. 5. Evolution of the transverse gradient of mean streamwise velocity with the separating streamlines plotted as dash lines 

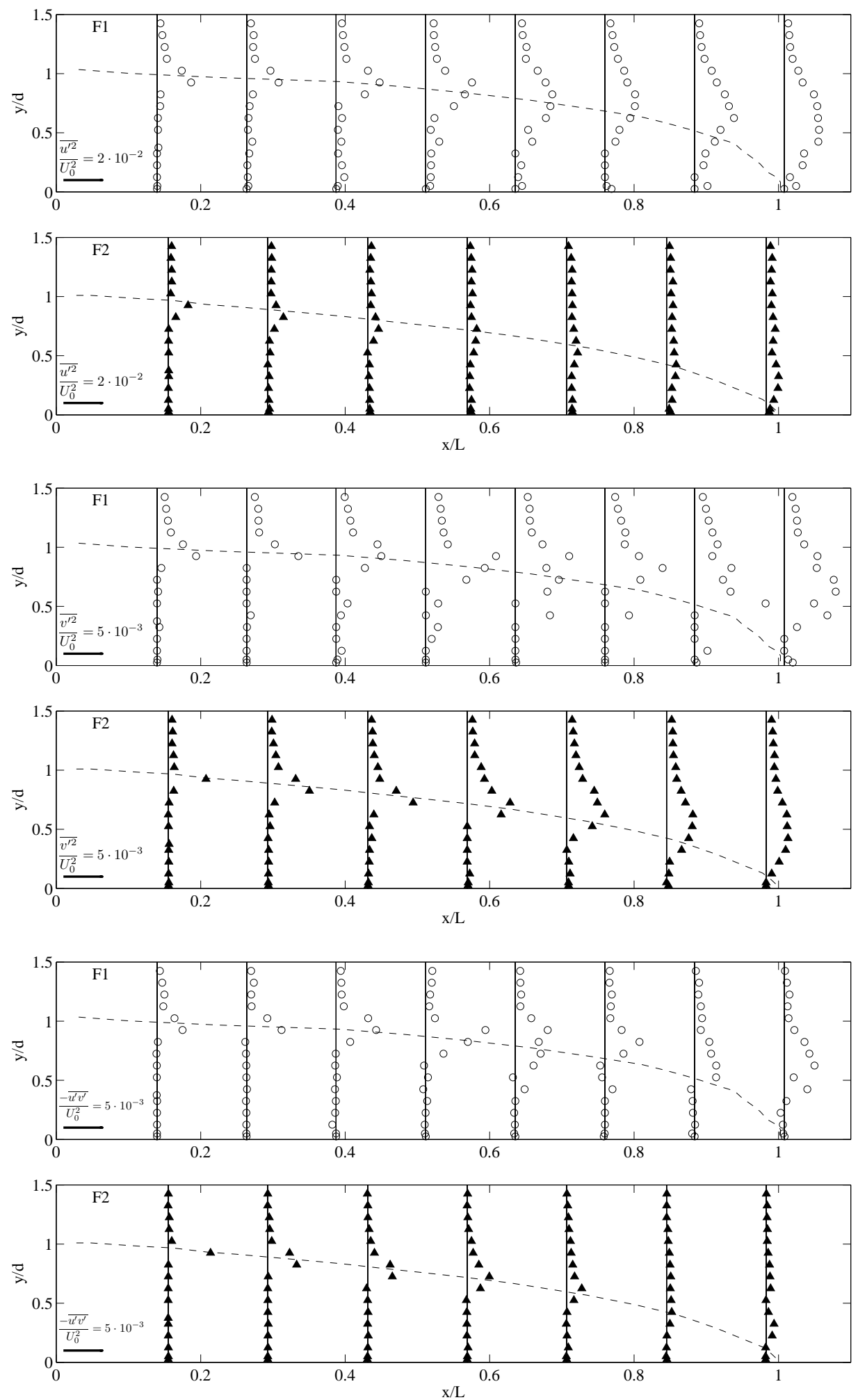

FIG. 6. Evolution of the Reynolds stress terms with the separating streamlines plotted as dash lines. Note: the different scales for the streamwise normal term and both other terms) 

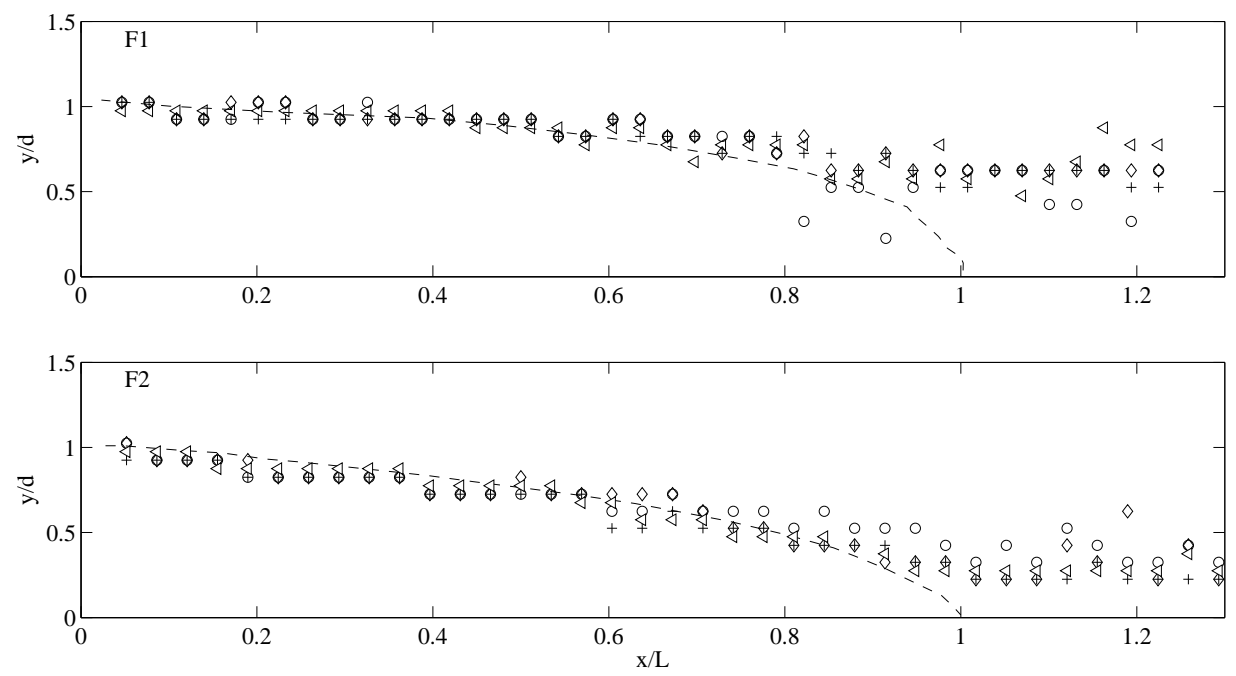

FIG. 7. Location of maximum velocity gradient $(\triangleleft), \overline{v^{\prime 2}}(\circ), \overline{v^{\prime}}(+)$ and $-\overline{u / v \prime}(\diamond)$ along with the separating streamline plotted as dash lines 


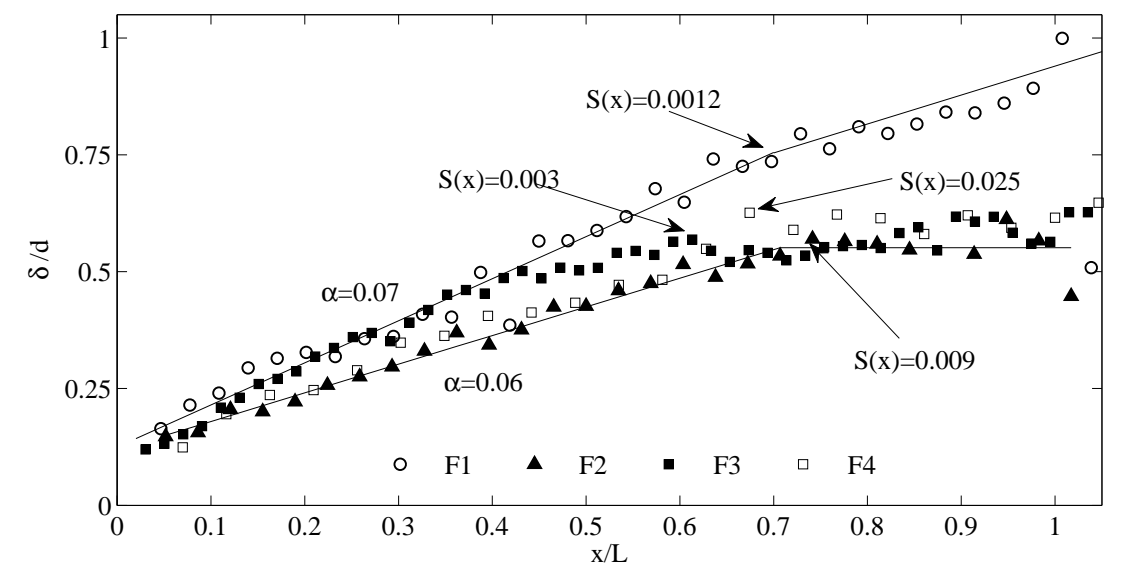

FIG. 8. Streamwise evolution of the measured (symbol) and fitted (plain lines -) non-dimensional mixing layer width 

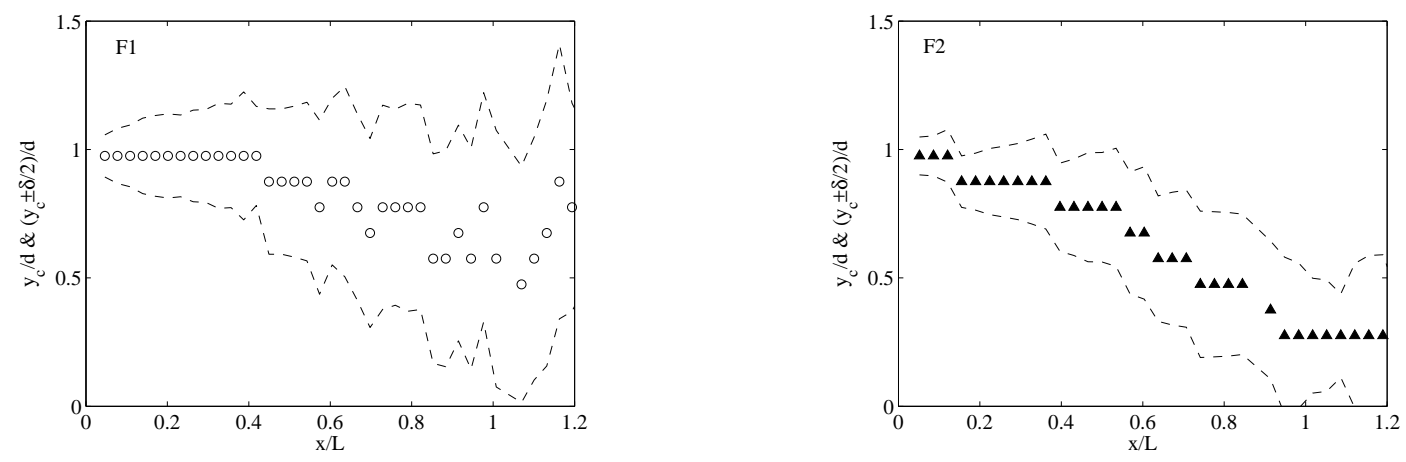

FIG. 9. Evolution of the mixing layer centerline (symbols) and the estimated mixing layer boundaries (dash lines --) 


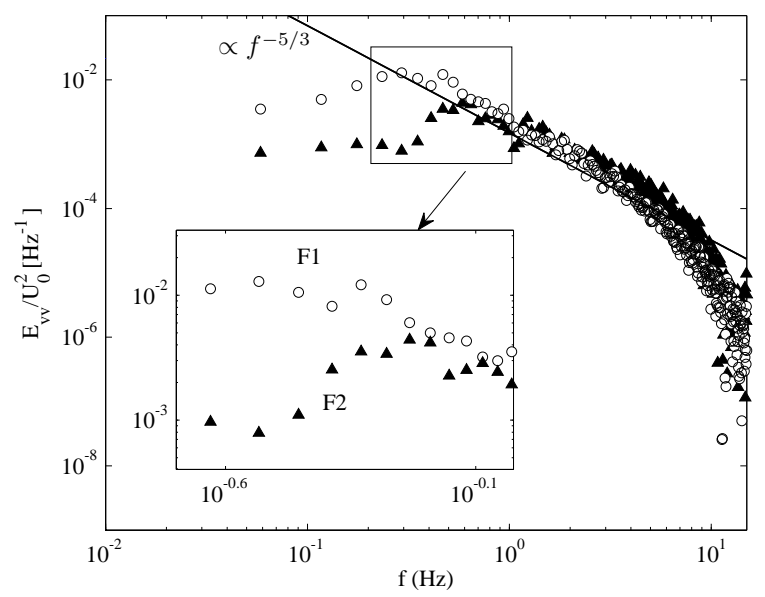

FIG. 10. Energy spectrum of the transverse velocity component $v$ along the centerline of the mixing layer at $x=0.2 \mathrm{~L}$. 

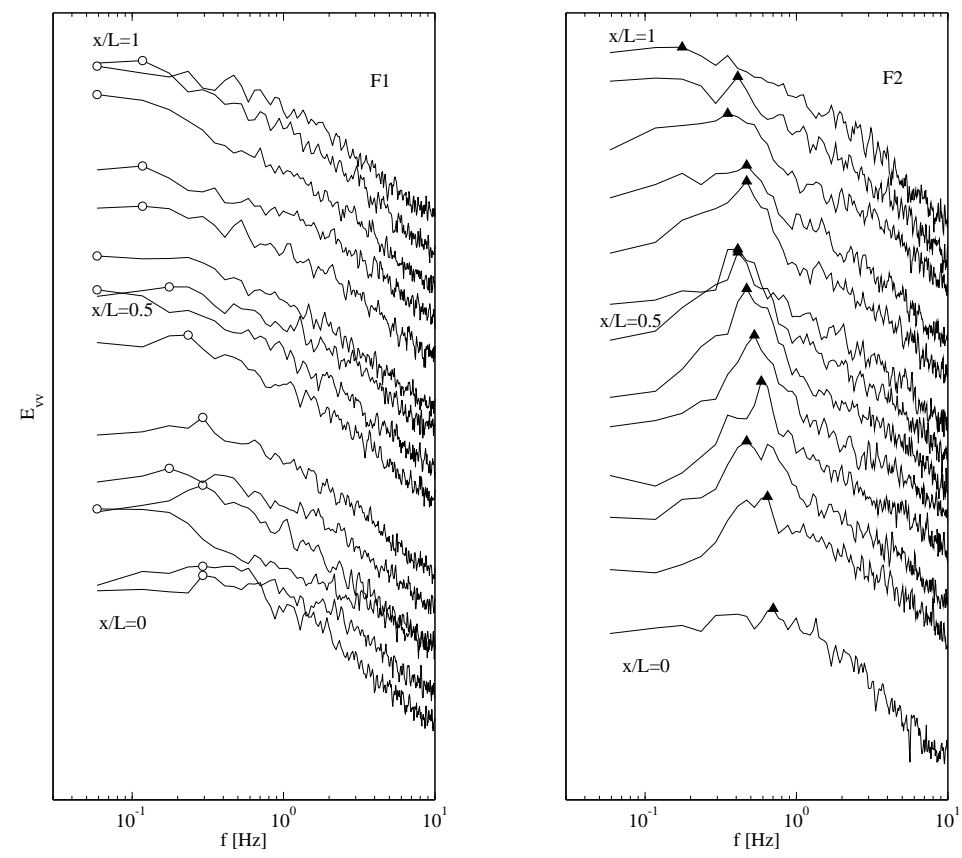

FIG. 11. Streamwise evolution of the energy spectrum of transverse velocity along the mixing layer centerline from $x / L=\mathbf{0}$ to $x / L=\mathbf{1}$. Note: the symbols presents the peak value of each $x / L$ section. 


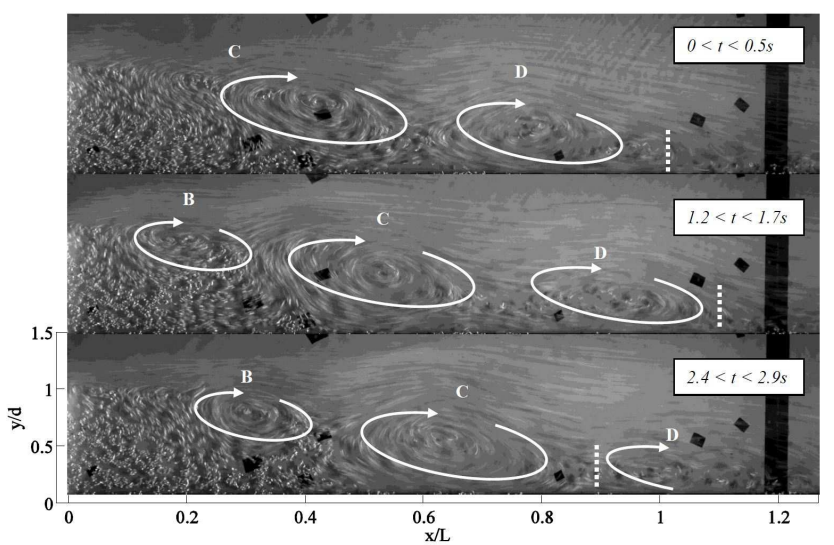

FIG. 12. Successive 0.5s exposure photographs for case F2. Dotted line : marks the location of the instantaneous reattachment 


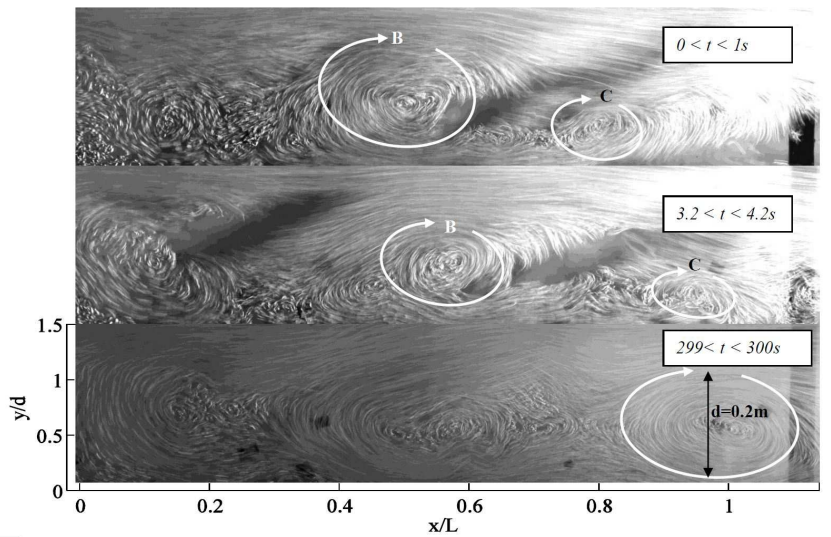

FIG. 13. Successive 1s exposure photographs for case F1 

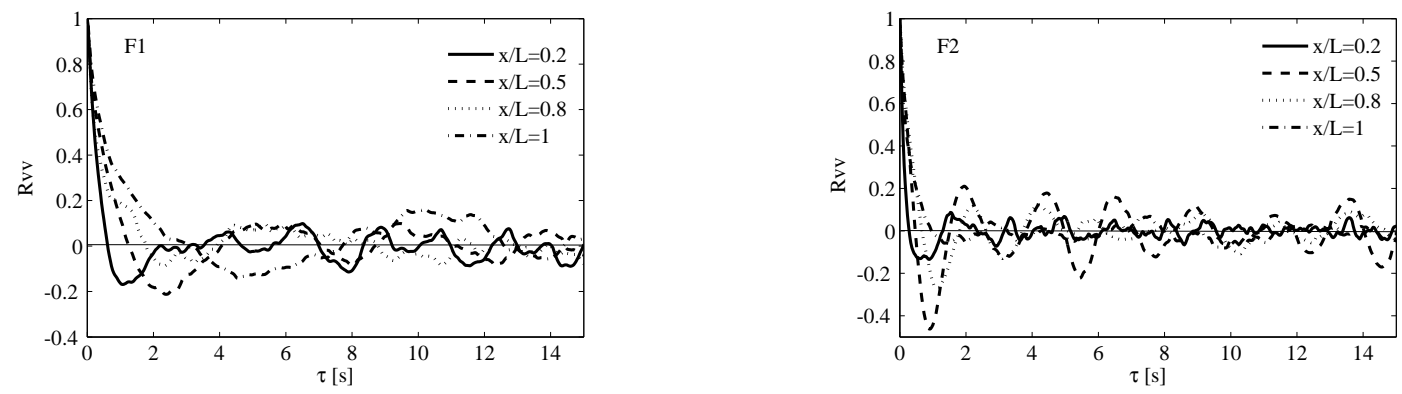

FIG. 14. Auto-correlation function of the transverse velocity fluctuation $R v v$ in different section of $x / L$ along the centerline of the mixing layer 


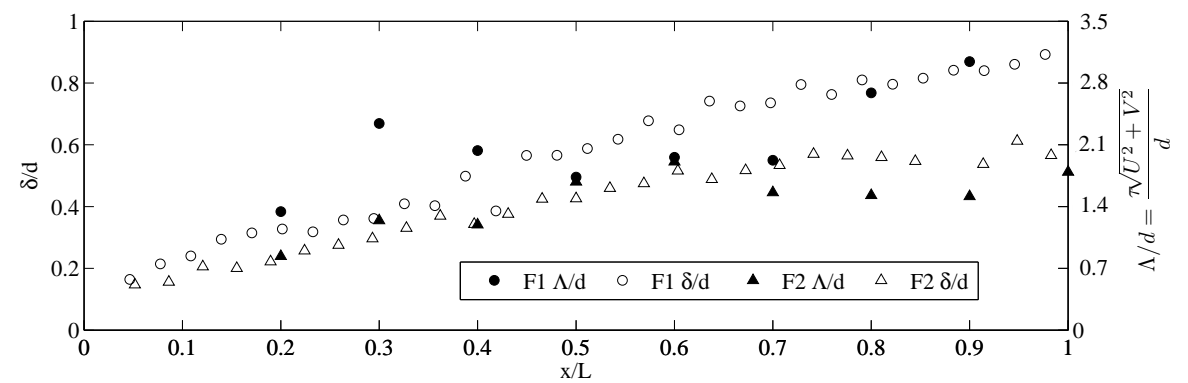

FIG. 15. The comparison between the mixing layer width (definition of Eq.2, left axis with symbols $\circ$ and $\triangle$ ) and longitudinal length scale of vortices (derived from the autocorrelation, right axis with symbols $\bullet$ and $\Delta$ ), along the mixing layer centerline from $x / L=\mathbf{0}$ to $x / L=1$ 